This is an author-created, un-copyedited version of an article accepted for publication/published in Modelling and Simulation in Materials Science and Engineering. IOP Publishing Ltd is not responsible for any errors or omissions in this version of the manuscript or any version derived from it. The Version of Record is available online at http://dx.doi.org/10.1088/0965-0393/24/6/065005. Citation details: Leyson, G. P. M.; Curtin, W. A. Model. Simul. Mater. Sci. Eng. 2016, 24 (6), 65005.

\title{
Solute strengthening at high temperatures
}

\author{
G.P.M. Leyson ${ }^{\mathrm{a}, *}$, W.A. Curtin ${ }^{\mathrm{b}}$ \\ ${ }^{a}$ Max-Planck-Institut für Eisenforschung GmbH, Düsseldorf 40237, Germany \\ ${ }^{b}$ Institute of Mechanical Engineering, Êcole Polytechnique Fédéral de Lausanne, Lausanne CH-1015, Switzerland
}

\begin{abstract}
The high temperature behavior of solute strengthening has previously been treated approximately using various scaling arguments, resulting in logarithmic and power-law scalings for the stressdependent energy barrier $\Delta E(\tau)$ versus stress $\tau$. Here, a parameter-free solute strengthening model is extended to high temperatures/low stresses without any a priori assumptions on the functional form of $\Delta E(\tau)$. The new model predicts that the well-established low-temperature, with energy barrier $\Delta E_{b}$ and zero temperature flow stress $\tau_{y 0}$, transitions to a near-logarithmic form for stresses in the regime $0.2<\tau / \tau_{y 0} \leq 0.5$ and then transitions to a power-law form at even lower stresses $\tau / \tau_{y 0}<0.03 . \Delta E_{b}$ and $\tau_{y 0}$ remains as the reference energy and stress scales over the entire range of stresses. The model is applied to literature data on solution strengthening in $\mathrm{Cu}$ alloys and captures the experimental results quantitatively and qualitatively. Most importantly, the model accurately captures the transition in strength from the low-temperature to intermediate-temperature and the associated transition for the activation volume. Overall, the present analysis unifies the different qualitative models in the literature and, when coupled with the previous parameter-free solute strengthening model, provides a single predictive model for solute strengthening as a function of composition, temperature, and strain rate over the full range of practical utility.
\end{abstract}

\section{Introduction}

Computational and theoretical materials science are powerful tools that can help develop design guidelines in the search for materials with improved performance. In particular, theoretical models that can give quantitative predictions at finite temperatures and experimental time scales provide the key connection between properties extracted from atomistic simulations and material behavior relevant in engineering applications [1-4]. Such models also provide a clear mechanistic understanding for observed macroscopic phenomena, and are able to replace phenomenology that does not have predictive capability.

One general domain of interest in metallurgy is that of "solute strengthening", i.e. the addition of solutes to a matrix to enhance the plastic flow/yield stress. Solute strengthening is a thermallyactivated process, and so finite-temperature/finite-strain rate solute strengthening is controlled by the energy barriers to dislocation motion, created by the solutes, that are overcome with the assistance of temperature and stress. The energy barrier $\Delta E(\tau)$ at a stress $\tau$ must thus be

${ }^{*}$ Current Address: Max-Planck-Straße 140237 Düsseldorf Germany Tel. No.: +49 (0)211 $6792-880$

Email addresses: g.leyson@mpie.de (G.P.M. Leyson), william.curtin@epfl.ch (W.A. Curtin) 
This is an author-created, un-copyedited version of an article accepted for publication/published in Modelling and Simulation in Materials Science and Engineering. IOP Publishing Ltd is not responsible for any errors or omissions in this version of the manuscript or any version derived from it. The Version of Record is available online at http://dx.doi.org/10.1088/0965-0393/24/6/065005. Citation details: Leyson, G. P. M.; Curtin, W. A. Model. Simul. Mater. Sci. Eng. 2016, 24 (6), 65005.

described accurately to achieve quantitative predictability. At low temperatures, for fcc materials, the stress-dependent energy barrier takes the general form [5],

$$
\Delta E(\tau)=\Delta E_{b}\left[1-\left(\frac{\tau}{\tau_{y 0}}\right)\right]^{3 / 2},
$$

where $\Delta E_{b}$ is the characteristic energy barrier, $\tau$ is the resolved shear stress and $\tau_{y 0}$ is the zerotemperature yield stress. A recent model gives parameter-free predictions for $\Delta E_{b}$ and $\tau_{y 0}$ as a function of solute types and solute concentrations $[6,7]$ that are in good agreement with various experimental results. Mechanistically, solute strengthening arises because the dislocation adopts a wavy character with some characteristic wavelength $\zeta_{c}$ and characteristic amplitude $w_{c}$ that allows the dislocation to find statistically-favorable regions of solute fluctuations that lower the overall energy of the dislocation. The random statistics of the solutes, the solute/dislocation interaction energies, and the dislocation line tension uniquely determine the scales $\zeta_{c}$ and $w_{c}$, from which $\Delta E_{b}$ and $\tau_{y 0}$ are derived (see Sec. 2).

With increasing temperature (corresponding to lower flow stresses), Labusch and also Zaiser note that the dislocation can become wavy on larger scales, finding even lower-energy configurations that have larger barriers that control the plastic flow. Labusch et al. estimated that $\Delta E(\tau)$ increases logarithmically with decreasing stress $\tau$ as $\Delta E(\tau) \propto \Delta E_{b} \ln \left(\tau_{y 0} / \tau\right)$ [8] while Zaiser proposed a power-law scaling of the energy $\Delta E(\tau) \propto \Delta E_{b}\left(\tau_{y 0} / \tau\right)^{\alpha}[9]$ with $1 / 7<\alpha<2 / 5$. Despite these scaling arguments, the high temperature/low stress behavior of $\Delta E(\tau)$ has never been explicitly calculated, never been related directly to the low-temperature energy and stress scales, nor have the regimes of "high temperature" or "low stress" been clearly quantified. In this paper, we extend our previous solute strengthening model $[6,7]$ valid at "low temperature/high stress" to the "high temperature/low stress" regime. We show that an analytic model predicts that the energy barrier can be well-approximated by a logarithmic form over the range $0.2<\tau / \tau_{y 0} \leq 0.5$ with a transition to a power-law form at even lower stresses $\tau / \tau_{y 0} \leq 0.03$, with $\Delta E_{b}$ as the reference energy across all regimes. We then show that the predictions of the new model are in good agreement with literature experiments, in particular capturing the transition in strength and activation volume with increasing temperature and/or decreasing strength.

\section{Solute strengthening at low-temperatures: single-scale bow-out}

We first review the major salient results of our solute strengthening model $[6,7]$ from which follow the new analyses that extend the model to high temperatures.

The input to the model is the solute/dislocation interaction energy $U\left(x_{i}, y_{j}\right)$ for a solute located at position $\left(x_{i}, y_{j}\right)$ relative to the center of the dislocation core, where $x$ is the glide direction, $y$ normal to the slip plane, and $z$ is the dislocation line direction. The model then envisions an initially straight dislocation of length $L$ exists in a field of randomly distributed solutes with concentration $c$. This dislocation is then able to bow out into a wavy configuration in the glide plane to lower its overall energy by residing in regions of where the statistical solute fluctuations lead to net binding of the solutes and the dislocation. The waviness has a cost, however, due to the increased dislocation line length, and so there is some optimal configuration. A general configuration of the dislocation is defined by a wavelength $\zeta$ and roughening amplitude $w$, and the the total energy change of the dislocation in this configuration compared to a straight 
This is an author-created, un-copyedited version of an article accepted for publication/published in Modelling and Simulation in Materials Science and Engineering. IOP Publishing Ltd is not responsible for any errors or omissions in this version of the manuscript or any version derived from it. The Version of Record is available online at http://dx.doi.org/10.1088/0965-0393/24/6/065005. Citation details: Leyson, G. P. M.; Curtin, W. A. Model. Simul. Mater. Sci. Eng. 2016, 24 (6), 65005.

dislocation is given by the elastic cost of bowing, proportional to the dislocation line tension $\Gamma$, and the potential energy gain due to interactions with favorable solute configurations,

$$
\Delta E_{t o t}(w, \zeta)=\left[\left(\Gamma \frac{w^{2}}{2 \zeta}\right)-\left(\frac{c \zeta}{\sqrt{3} b}\right)^{\frac{1}{2}} \Delta \tilde{E}_{p}(w)\right] \cdot\left(\frac{L}{2 \zeta}\right) .
$$

The term $\Delta \tilde{E}_{p}(w)$ is the change in energy, per unit length, of a straight segment of dislocation as it moves a distance $w$ through the solute field,

$$
\Delta \tilde{E}_{p}(w)=\left[\sum_{i j}\left(U\left(x_{i}-w, y_{j}\right)-U\left(x_{i}, y_{j}\right)\right)^{2}\right]^{\frac{1}{2}} .
$$

The characteristic dislocation wavelength $\zeta_{c}$ and amplitude $w_{c}$ are found by minimizing the total energy of the dislocation line. Minimizing Eqn. (2) with respect to $\zeta$ yields

$$
\zeta_{c}(w)=\left(4 \sqrt{3} \frac{\Gamma^{2} w^{4} b}{c \Delta \tilde{E}_{p}^{2}(w)}\right)^{\frac{1}{3}}
$$

Substituting Eqn. (4) into Eqn. (2), and then minimizing with respect to $w$ gives the characteristic roughening amplitude $w_{c}$ and characteristic energy $\Delta E_{c}=\Delta E_{t o t}\left(w_{c}, \zeta_{c}\left(w_{c}\right)\right) /\left(2 \zeta_{c} / L\right)$; this minimization must usually be performed numerically.

The characteristic energy barrier $\Delta E_{b}$ for the trapped dislocation segments of length $\zeta_{c}$ is related to the characteristic energy $\Delta E_{c}$ as (see Ref. [7])

$$
\Delta E_{b}=\left(\frac{4 \sqrt{2}-1}{3} \cdot \frac{3^{5 / 6}}{2^{5 / 3}}\right)\left(\frac{c w_{c}^{2} \Gamma \Delta \tilde{E}_{p}^{2}\left(w_{c}\right)}{b}\right)^{\frac{1}{3}}
$$

For a locally-sinusoidal energy landscape with barrier height $\Delta E_{b}$ located $w_{c}$ away from the minimum, the work done on the dislocation segment by an applied stress $\tau$ over a distance $x$ tilts the energy landscape and reduces the barrier. At a critical stress, the barrier becomes zero, so that flow requires no thermal activation. This critical stress is the zero-temperature yield stress, and is given by

$$
\tau_{y 0}=\frac{\pi}{2} \frac{\Delta E_{b}}{b \zeta_{c}\left(w_{c}\right) w_{c}}=1.01\left(\frac{c^{2} \Delta \tilde{E}_{p}^{4}\left(w_{c}\right)}{\Gamma b^{5} w_{c}^{5}}\right)^{\frac{1}{3}},
$$

The stress-dependent energy barrier for this energy landscape can then be written in the form of Eq. (1). Using Orowan's law and an Arrhenius model for the thermal activation, the finitetemperature, finite-strain-rate yield stress $\tau$ is then obtained by solving

$$
\Delta E(\tau)=k_{B} T \ln \frac{\dot{\epsilon}_{\circ}}{\dot{\epsilon}}
$$

where $k_{B}$ is the Boltzmann constant, $T$ is the temperature, $\dot{\epsilon}$ is the strain rate and $\dot{\epsilon}_{\circ}$ is the reference strain rate, typically ranging from $10^{4}-10^{6} \mathrm{~s}^{-1}$. This completes the general theory for solute strengthening. 
This is an author-created, un-copyedited version of an article accepted for publication/published in Modelling and Simulation in Materials Science and Engineering. IOP Publishing Ltd is not responsible for any errors or omissions in this version of the manuscript or any version derived from it. The Version of Record is available online at http://dx.doi.org/10.1088/0965-0393/24/6/065005. Citation details: Leyson, G. P. M.; Curtin, W. A. Model. Simul. Mater. Sci. Eng. 2016, 24 (6), 65005.

Specific applications of the model require the solute/dislocation interaction energy versus solute position, $U\left(x_{i}, y_{j}\right)$. For simplicity and generality, in this paper we use only the elastic interaction energy due to the interaction of the solute misfit volume $\Delta v_{m}$ with the dislocation pressure field $p\left(x_{i}, y_{j}\right), U\left(x_{i}, y_{j}\right)=-p\left(x_{i}, y_{j}\right) \Delta v_{m}$. We also use a simple spread-core model for the dissociated edge dislocation in an fcc matrix. The solute-dislocation interaction energy is then given by

$$
U\left(x_{i}, y_{j}\right)=-\frac{(1+\nu) \mu}{3 \pi(1-\nu)} \frac{b}{n+1} \sum_{l=-n / 2}^{n / 2} \frac{y_{j} \Delta v_{m}}{\left[x_{i}-l\left(\frac{b}{2}\right)\right]^{2}+y_{j}^{2}},
$$

where $\nu$ is the Poisson ratio, $\mu$ is the shear modulus, $b$ is the Burgers vector, and the core width is $n=10$ corresponding to the core width in aluminum. The general results of this paper are unchanged when more-detailed descriptions of the solute-dislocation interaction energy (i.e., considering the chemical interaction between solutes and the stacking fault, using a PeierlsNabarro description of the dislocation core) are used.

An important additional feature of the above model is that the minimization of the total energy of dislocation in a random solute field may have multiple solutions. Whether or not this occurs depends on the details of the solute/dislocation interactions and including the dislocation structure. In an $\mathrm{Al}$ matrix, only one solution is found. For basal slip in $\mathrm{Mg}$, however, where the low stacking fault energy leads to a wider dissociation distance of the two partial dislocations, two solutions emerge. One solution has a high strength and low energy barrier, and controls the low-T strengthening, while the other solution has a lower strength but higher energy barrier and controls the strength at high-T. One cannot determine how many solutions exist without precise results. Here, we focus on the low-T solution and extend the analysis to higher temperatures but recognize that the existence of other energy-minimizing solutions could also contribute or determine the strengthening at higher temperatures.

\section{Solute-strengthening at elevated temperatures: multiscale bow-out}

The previous analysis minimizes the total energy of the dislocation by allowing it to bow out with a single set of length scales $w$ and $\zeta$. However, the dislocation can reduce its energy further by bowing out over multiple length scales, and thus taking advantage of larger-scale solute fluctuations. Fig. 1 shows a schematic of the multiscale bowout, using a simple sinusoid for the wavy dislocation at each scale. To start, consider that the dislocation can bow out over two length scales $\zeta_{1}$ and $\zeta_{2}$. We assume that the two scales are well separated (i.e. $\zeta_{2} \gg \zeta_{1}$ ), such that the total line length is well-approximated by the sum of the two line lengths. The change in total energy has contributions from both scales [10],

$$
\Delta E_{\text {tot }}=\Delta E_{1}^{\text {bow }}\left(w_{1}, \zeta_{1}\right)+\Delta E_{2}^{\text {tot }}\left(w_{2}, \zeta_{2}\right)
$$

The contribution at the smallest scale, $\Delta E_{1}^{\text {bow }}\left(w_{1}, \zeta_{1}\right)$, is precisely the low-temperature theory, with minimized energy at $w_{c, 1}=w_{c}$ and $\zeta_{c, 1}=\zeta_{c}$. The additional energy at the second scale again has two terms. There is an additional line length $\left(w_{2}-w_{c, 1}\right)^{2} / 2 \zeta_{2}$ per $2 \zeta_{2}$ that costs elastic energy according to the line tension. There is also, however, an additional potential energy corresponding to larger-scale solute fluctuations. This energy should not include the energy of fluctuations already contained at the smaller scale $w_{c, 1}$, however. Therefore, the energy 
This is an author-created, un-copyedited version of an article accepted for publication/published in Modelling and Simulation in Materials Science and Engineering. IOP Publishing Ltd is not responsible for any errors or omissions in this version of the manuscript or any version derived from it. The Version of Record is available online at http://dx.doi.org/10.1088/0965-0393/24/6/065005. Citation details: Leyson, G. P. M.; Curtin, W. A. Model. Simul. Mater. Sci. Eng. 2016, 24 (6), 65005.

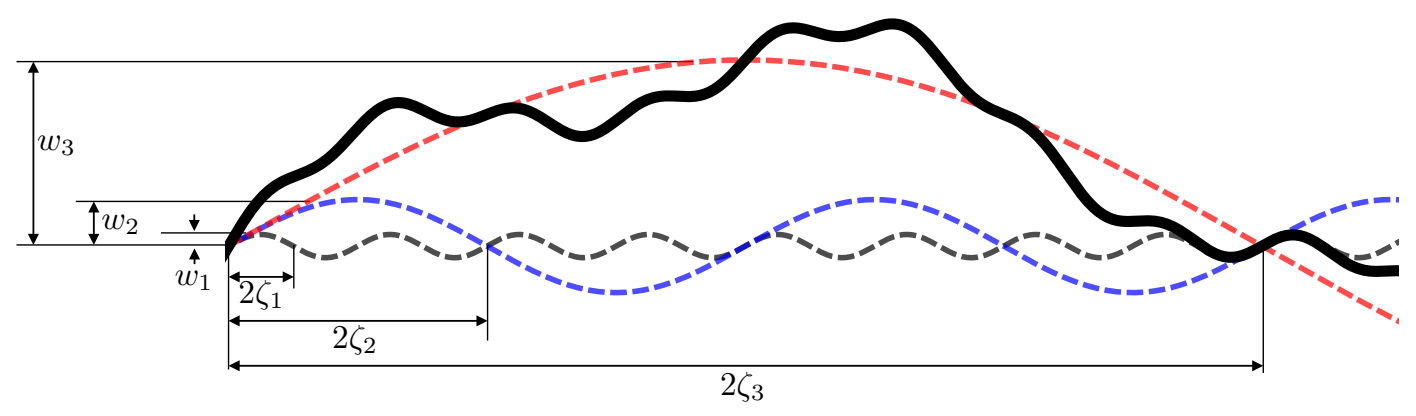

Figure 1: Schematic representation of the multi-scale bow-out configuration (bold black line) for three scales of bow-out (dashed lines), using a simple sinusoid to represent the bow-out at each scale.

contribution $\Delta E_{2}^{\text {tot }}\left(w_{2}, \zeta_{2}\right)$ is

$$
\Delta E_{2}^{\mathrm{bow}}\left(w_{2}, \zeta_{2}\right)=\left[\left(\Gamma \frac{\left(w_{2}-w_{c, 1}\right)^{2}}{2 \zeta_{2}}\right)-\left(\frac{c \zeta_{2}}{\sqrt{3} b}\right)^{\frac{1}{2}}\left(\Delta \tilde{E}_{p}\left(w_{2}\right)-\Delta \tilde{E}_{p}\left(w_{c, 1}\right)\right)\right] \cdot\left(\frac{L}{2 \zeta_{2}}\right) .
$$

Generalizing the above result, the dislocation can bow out over an arbitrary number of scales (e.g. Fig. 1). The total energy change can then be expressed as $\Delta E_{\text {tot }}=\sum_{i} \Delta E_{i}^{\text {bow }}$ where

$$
\Delta E_{i}^{\text {bow }}\left(w_{i}, \zeta_{i}\right)=\left[\left(\Gamma \frac{\left(w_{i}-w_{c, i}\right)^{2}}{2 \zeta_{i}}\right)-\left(\frac{c \zeta_{i}}{\sqrt{3} b}\right)^{\frac{1}{2}}\left(\Delta \tilde{E}_{p}\left(w_{i}\right)-\Delta \tilde{E}_{p}\left(w_{c, i-1}\right)\right)\right] \cdot\left(\frac{L}{2 \zeta_{i}}\right) .
$$

with $w_{c, 0}=0$ and $\Delta \tilde{E}_{p}\left(w_{0}\right)=0$. The total dislocation energy is minimized by minimizing the additional energy at each successive bow-out scale, i.e. minimizing Equation (11) at fixed $w_{c, i-1}, \zeta_{c, i-1}$ to give the characteristic length scales $w_{c, i}, \zeta_{c, i}$. The resulting relationship between $\zeta_{c, i}$ and $w_{c, i}$ is shown in Fig. 2, and approximately follows a power-law. Fig. 2 shows the first four length scales but in practice only the first few configurations are relevant at temperatures below the metal melting point. We note that truncating the solute/dislocation interaction energies would limit the number of bowout scales, and so the robust scaling here emerges from considering the long-range nature of the solute/dislocation interactions. Generalizing the scaling to continuous values of $w$ and $\zeta$, the power-law is

$$
\frac{\zeta(w)}{\zeta_{c}}=\left(\frac{w}{w_{c}}\right)^{\phi}
$$

where $w_{c}$ and $\zeta_{c}$ now refer exclusively to the lowest scale, low-temperature values. The roughening exponent $\phi=1.47$ is very close to the value $3 / 2$ estimated in previous literature for related but not identical problems $[9,11]$ and using very different scaling arguments.

The above analysis shows that the dislocation can minimize its total energy by adopting multiple bowouts at scales $\left(w_{c, i}, \zeta_{c, i}\right)$, However, to reach the larger-scale bowout configurations requires overcoming increasingly large energy barriers; this was a key general insight by Labusch that we quantify within the context of our model. Specifically, kinetic considerations prevent the dislocation from reaching the successive configurations corresponding to the global energy 
This is an author-created, un-copyedited version of an article accepted for publication/published in Modelling and Simulation in Materials Science and Engineering. IOP Publishing Ltd is not responsible for any errors or omissions in this version of the manuscript or any version derived from it. The Version of Record is available online at http://dx.doi.org/10.1088/0965-0393/24/6/065005. Citation details: Leyson, G. P. M.; Curtin, W. A. Model. Simul. Mater. Sci. Eng. 2016, 24 (6), 65005.

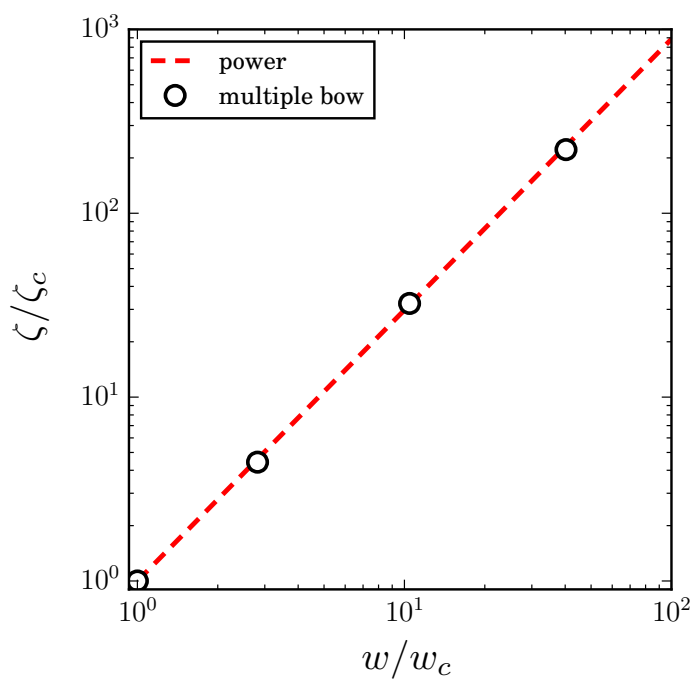

Figure 2: Characteristic wavelength $\zeta_{c, i}$ versus characteristic roughening amplitude $w_{c, i}$ for the multiple bow-out model (solid symbols). Dashed line: power law fit with roughening coefficient $\phi=1.47$.

minima. However, the dislocation can lower its total energy by bowing out over kineticallyaccessible additional length scale(s), even if it is unable to reach the global minimum. We now pursue this idea quantitatively.

Following the general analysis above, we now consider two-level bow-out with the second level having a continuous range of possible bow out scales. That is, we consider bow-out at $w_{c, 1}=w_{c}$, $\zeta_{c, 1}=\zeta_{c}$ and at a second level $w>w_{c}$ and $\zeta>\zeta_{c}$. At any $w>w_{c}$, we minimize the energy with respect to $\zeta$ by using the scaling relation of Eqn. (12) for $\zeta(w)$. The additional energy reduction by adding this second scale is

$$
\Delta E_{2}^{\text {bow }}\left(w_{2}, \zeta_{2}\right)=\left[\left(\Gamma \frac{\left(w_{2}-w_{c}\right)^{2}}{2 \zeta_{2}}\right)-\left(\frac{c \zeta_{2}}{\sqrt{3} b}\right)^{\frac{1}{2}}\left(\Delta \tilde{E}_{p}\left(w_{2}\right)-\Delta \tilde{E}_{p}\left(w_{c}\right)\right)\right] \cdot\left(\frac{L}{2 \zeta_{2}}\right) .
$$

Figure 3 shows that indeed the energy is reduced steadily with increasing $w_{2}$ until the energy reaches the minimum at $w_{2}=w_{c, 2}$. The dislocation therefore evolves to increase $w_{2}$, with $\zeta_{2}$ evolving according to the scaling law, and minimizes its total energy.

However, exploring increasing $w_{2}$ requires overcoming energy barriers. For bow-out at a given $\left(\zeta_{2}, w_{2}\right)$, the corresponding energy barrier and zero-temperature strength are

$$
\Delta E_{b}\left(w_{2}\right)=\left(\frac{2 c \zeta\left(w_{2}\right)}{\sqrt{3} b}\right)^{\frac{1}{2}} \Delta \tilde{E}_{p}\left(w_{2}\right)-\left(\Gamma \frac{w_{2}^{2}}{2 \zeta\left(w_{2}\right)}\right)
$$

and

$$
\tau_{y 0}\left(w_{2}\right)=\frac{\pi}{2} \frac{\Delta E_{b}\left(w_{2}\right)}{b \zeta\left(w_{2}\right) w_{2}} .
$$

At a given applied stress $\tau$, the evolution of the dislocation toward larger $w_{2}$ is impeded by the maximum barrier encountered. Flow, i.e. motion of the dislocation, is then controlled by the rate at which the dislocation can overcome this maximum barrier. At a given $\tau$, the barrier 
This is an author-created, un-copyedited version of an article accepted for publication/published in Modelling and Simulation in Materials Science and Engineering. IOP Publishing Ltd is not responsible for any errors or omissions in this version of the manuscript or any version derived from it. The Version of Record is available online at http://dx.doi.org/10.1088/0965-0393/24/6/065005. Citation details: Leyson, G. P. M.; Curtin, W. A. Model. Simul. Mater. Sci. Eng. 2016, 24 (6), 65005.

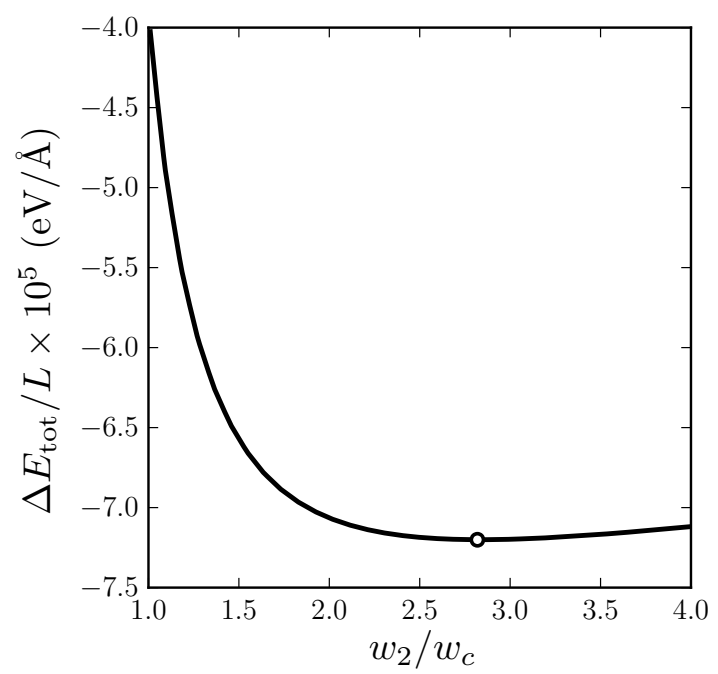

Figure 3: Total energy change of a dislocation versus bow-out amplitude at a second scale, for two-scale bow-out with the characteristic length $\zeta_{2}$ determined by Eqn. (12). Open circle: minimum energy point at $w_{c}=w_{c, 2}$.

versus $w_{2}$ is computed using the above equations and the results are shown in Figure 4 , with the maximum barrier at each stress level indicated. At high stresses $\tau / \tau_{y 0}>0.5$, the maximum barrier is at $w_{c}$, i.e. the single-level bow-out solution determines the flow behavior. Although the total energy of the dislocation could be decreased by introducing $w_{2}$, the dislocation is only able to move over the original barrier at scale $w_{c}$ and so cannot access the larger fluctuations. With decreasing stress, the maximum barrier occurs at increasing $w_{2}>w_{c}$. Thus, at lower stresses, the dislocation adopts a second scale of waviness that lowers the total energy, but increases the energy barrier for flow. Once $w_{2}=w_{c, 2}$, the dislocation can then bow-out at a third scale $w_{3}$, further minimizing the total energy but kinetically limited by an ever-increasing energy barrier; the analysis is similar to that presented just above. The theory here thus provides a quantitative calculation of the controlling energy barrier as a function of the stress $\tau$.

By considering a continuous range of stresses $\tau$, we can compute the maximum, controlling, barrier as a function of $\tau$, as shown in Fig. 5. Consistent with Fig. 4, the energy barrier is monotonically increasing with decreasing stress. For reference, Fig. 5 also shows the energy barrier for the single-scale bow-out model, which is valid at high stresses $\left(\tau / \tau_{y 0}>0.5\right)$. Thus, as postulated in our original work, the single-scale minimum total energy configuration for the wavy dislocation corresponds to the largest barrier at stresses near the zero-temperature yield stress $\tau_{y 0}$. However, we now see that the barrier increases even faster at lower stresses, with deviations from the low-temperature/high-stress model at $\tau / \tau_{y 0}<0.5$.

Examining the multiscale bow-out prediction in Fig. 5, we find that the energy barrier versus stress can be well-approximated by a logarithmic relationship

$$
\Delta E(\tau)=C_{l} \Delta E_{b} \ln \left(\frac{\tau_{y 0}}{\tau}\right)
$$

at intermediate stresses $0.2<\tau / \tau_{y 0}<0.5$ with the constant $C_{l}=0.55$. Here, and for the rest of this paper, $\Delta E_{b}$ and $\tau_{y 0}$ refer to the characteristic energy barrier and the zero-temperature yield stress of the minimum energy single-scale bow-out configuration. As mentioned earlier, 
This is an author-created, un-copyedited version of an article accepted for publication/published in Modelling and Simulation in Materials Science and Engineering. IOP Publishing Ltd is not responsible for any errors or omissions in this version of the manuscript or any version derived from it. The Version of Record is available online at http://dx.doi.org/10.1088/0965-0393/24/6/065005. Citation details: Leyson, G. P. M.; Curtin, W. A. Model. Simul. Mater. Sci. Eng. 2016, 24 (6), 65005.

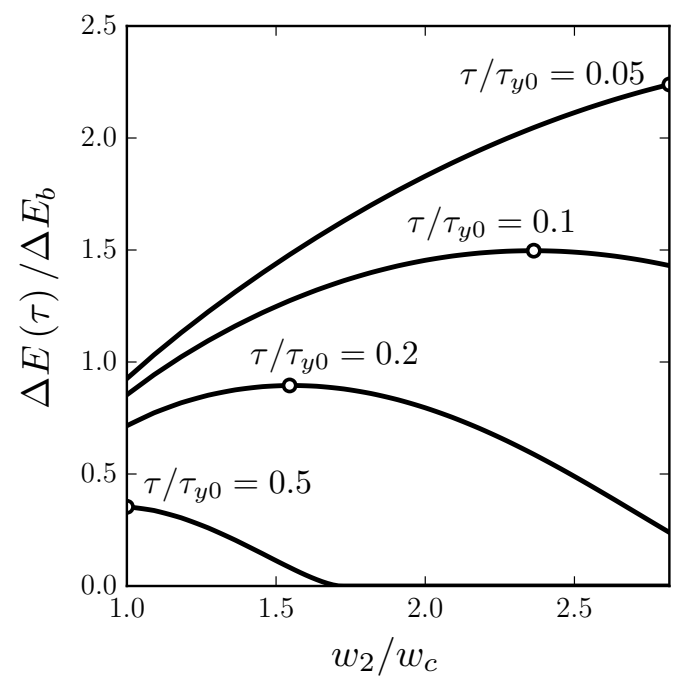

Figure 4: Energy barrier $\Delta E(\tau)$ versus second-scale bow-out amplitude $w_{2}$, for various applied stresses. Open circles: maximum energy barrier at each stress.

the logarithmic form was first proposed by Labusch [8] with a similar physical rationale but less quantification. The value $C_{l}=0.55$ differs slightly from the value 0.51 we have used previously $[6,7,12]$ because in the earlier work we simply assumed a Labusch-type model and fit a logarithmic model to match the low-temperature model over the range $0.3<\tau / \tau_{y 0}<0.6$. The new value of 0.55 provides an accurate fit to our new derived model in the range of $0.2<\tau / \tau_{y 0}<0.5$, where the low temperature solution begins to fail. Using the logarithmic model with coefficient 0.55 at larger stresses $0.5<\tau / \tau_{y 0}$ slightly overestimates the barrier and thus overestimates the strength relative to the accurate low-temperature predictions, but only by at most $6.4 \%$ so that the logarithmic form with $C_{l}=0.55$ can provide a single model with good accuracy over a wide range of stress.

Furthermore, at lower stresses $\left(\tau / \tau_{y 0}<0.2\right)$, the logarithmic model underestimates the energy barrier predicted by the full multiscale bow-out model, which predicts a gradual transition to a power-law scaling. At stresses $\left(\tau / \tau_{y 0}<0.03\right)$, the transition to a power-law is essentially complete and the scaling of the energy barrier can be well-approximated as

$$
\Delta E(\tau)=C_{p} \Delta E_{b}\left(\frac{\tau_{y 0}}{\tau}\right)^{\varphi}
$$

where $C_{p}=0.52$ and $\varphi=0.54$. A similar power-law relationship was proposed by Zaiser [9] using scaling arguments for the relationship between $w$ and $\zeta$. However, Zaiser only estimated the power-law exponent as $\varphi=0.25$. Here, the power-law form emerges as the very low-stress limit of a multiscale bow-out analysis.

Overall, the new multiscale bow-out model reconciles all the functional forms for solid solution strengthening versus temperature proposed in the literature and clearly identifies the regimes where each form is valid/useful. The analysis also demonstrates that the energy barrier and strength scale with the single-scale bow-out model energy barrier $E_{b}$ and zero-temperature yield stress $\tau_{y 0}$. Therefore, predictions over the entire range of stress and temperature emerge from quantities computed in the original predictive solute strengthening model. 
This is an author-created, un-copyedited version of an article accepted for publication/published in Modelling and Simulation in Materials Science and Engineering. IOP Publishing Ltd is not responsible for any errors or omissions in this version of the manuscript or any version derived from it. The Version of Record is available online at http://dx.doi.org/10.1088/0965-0393/24/6/065005. Citation details: Leyson, G. P. M.; Curtin, W. A. Model. Simul. Mater. Sci. Eng. 2016, 24 (6), 65005.

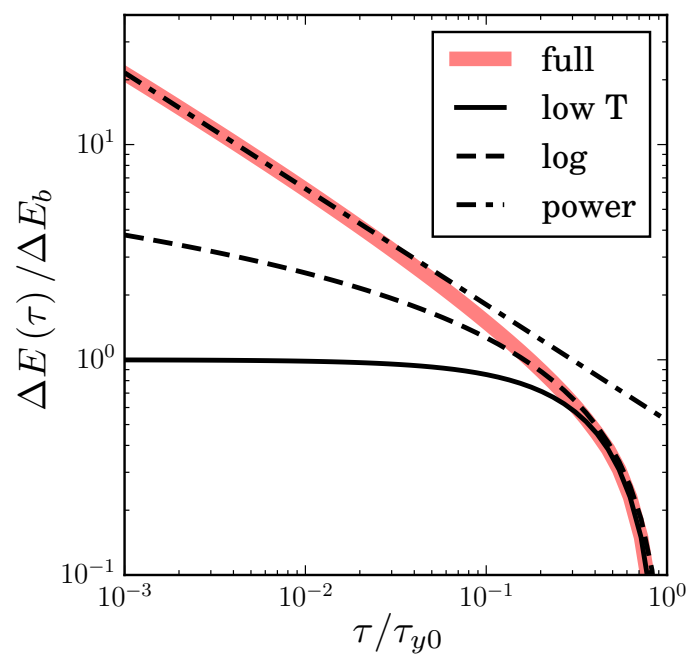

Figure 5: Energy barrier $\Delta E(\tau)$ versus applied stress $\tau$. Red line: full multiscale bow-out model; Black line: low temperature model; Dashed line: intermediate temperature model (logarithmic form); Dashed-dot line: high temperature model (power law form).

\section{Solute strengthening at high temperatures: predictions}

We now demonstrate that the predictions of the multiscale bow-out model explain detailed trends in the long-standing experimental literature on solid solution strengthening. Experiments measure the yield stress and/or the activation volume as a function of the temperature, the imposed strain rate, and the alloy composition. The full solute strengthening theory provides values for the concentration-scaled energy barrier $E_{b} / c^{1 / 3}$ and concentration-scaled zero-temperature yield strength $\tau_{y 0} / c^{2 / 3}$ for a matrix with a concentration $c$ of one solute type. As noted earlier, we envision only one solution, and thus one combination of $\left(E_{b} / c^{1 / 3}, \tau_{y 0} / c^{2 / 3}\right)$, being predicted by the model. Some materials may have multiple solutions that further affect the strength at higher T. Generalization to multiple solutes is straightforward but here we consider binary alloys only.

Given the energy barrier and zero- $T$ strength, we use the standard Arrhenius model as in Section 2 to compute desired quantities. The full solution over the entire temperature range is numerically calculated. The analytic functional forms permit approximate analytic solutions over the relevant domains of applicability. Specifically, using Eqns. (7), (16), and (17), we obtain the approximate finite temperature yield stress as

$$
\tau_{y}(T)= \begin{cases}\tau_{y 0}\left[1-\left(\frac{k_{B} T}{\Delta E_{b}} \ln \frac{\dot{\epsilon_{\circ}}}{\dot{\epsilon}}\right)^{\frac{2}{3}}\right] & \tau_{y} / \tau_{y 0}>0.5, \\ \tau_{y 0} \exp \left(-\frac{1}{C_{1}} \frac{k_{B} T}{\Delta E_{b}} \ln \frac{\dot{\epsilon_{\circ}}}{\dot{\epsilon}}\right) & 0.2<\tau_{y} / \tau_{y 0}<0.5, \\ \tau_{y 0}\left(\frac{1}{C_{\mathrm{p}}} \frac{k_{B} T}{\Delta E_{b}} \ln \frac{\dot{\epsilon_{\circ}}}{\dot{\epsilon}}\right)^{-\frac{1}{\varphi}} & \tau_{y} / \tau_{y 0}<0.03,\end{cases}
$$

The activation volume $V=-\partial \Delta E / \partial \tau$ is obtained by taking the indicated derivatives with respect 
This is an author-created, un-copyedited version of an article accepted for publication/published in Modelling and Simulation in Materials Science and Engineering. IOP Publishing Ltd is not responsible for any errors or omissions in this version of the manuscript or any version derived from it. The Version of Record is available online at http://dx.doi.org/10.1088/0965-0393/24/6/065005. Citation details: Leyson, G. P. M.; Curtin, W. A. Model. Simul. Mater. Sci. Eng. 2016, 24 (6), 65005.

Table 1: Normalized characteristic energy barrier and normalized zero temperature yield stress for various copper alloys, as obtained by fitting to experiment for each alloy, $\mathrm{Cu}-\mathrm{Al}$ [13]; $\mathrm{Cu}-\mathrm{Ge}$ and $\mathrm{Cu}-\mathrm{Mn}$ [14].

\begin{tabular}{|c|ccc|}
\hline Solute & $\mathrm{Al}$ & $\mathrm{Ge}$ & $\mathrm{Mn}$ \\
\hline$\Delta E_{b} / c^{1 / 3}(\mathrm{eV})$ & 3.96 & 3.02 & 4.11 \\
$\tau_{y 0} / c^{2 / 3}(\mathrm{MPa})$ & 187.3 & 227.2 & 416.8 \\
\hline
\end{tabular}

to $\tau$. For the approximate analytic models, the activation volumes are

$$
V\left(\tau_{y}, T\right)= \begin{cases}\frac{3}{2} \frac{\Delta E_{b}}{\tau_{y 0}}\left(\frac{k_{B} T}{\Delta E_{b}} \ln \frac{\dot{\epsilon}_{\circ}}{\dot{\epsilon}}\right)^{\frac{1}{3}} & \tau_{y} / \tau_{y 0}>0.5, \\ C_{l} \Delta E_{b} \tau_{y}^{-1} & 0.2<\tau_{y} / \tau_{y 0}<0.5, \\ \left(\varphi k_{B} T \ln \frac{\dot{\epsilon}_{\circ}}{\dot{\epsilon}}\right) \tau_{y}^{-1} & \tau_{y} / \tau_{y 0}<0.03,\end{cases}
$$

The transition from logarithmic to power-law form in the stress range $0.03<\tau / \mid$ tau $u_{y 0}<0.2$ can be approximated by some interpolation; Fig. 5 indicates the differences between the approximate and numerical results.

We now validate the model by comparing predictions to experimental data on copper alloys containing $\mathrm{Al}$ [13], Ge and $\mathrm{Mn}$ [14] at various solute concentrations and temperatures. We do not have all of the solute/dislocation interaction energies necessary to make full parameter-free predictions, but this is not crucial since we are interested in the behavior versus temperature and yield stress. Therefore, for each type of solute, the normalized characteristic energy barrier $\Delta E_{b} / c^{1 / 3}$ and the normalized zero temperature yield stress $\tau_{y 0} / c^{2 / 3}$ are used as fitting parameters obtained by a least-squares fit to all of the measured strengths over a range of compositions and temperatures. The Peierls stress of pure $\mathrm{Cu}$ is neglected as small, reported by Basinski to be $0.198 \mathrm{MPa}[13]$. The values obtained for the normalized quantities range from 187 to $416 \mathrm{MPa}$ for $\tau_{y 0} / c^{2 / 3}$ and 3.02 to $4.11 \mathrm{eV}$ for $\Delta E_{b} / c^{1 / 3}$, and are summarized in Table 1 . These are roughly comparable to the values we have obtained for entirely different systems. For Al-X alloys $(\mathrm{X}=\mathrm{Mg}$, $\mathrm{Cu}, \mathrm{Cr}, \mathrm{Mn}), \tau_{y 0} / c^{2 / 3}=137-711 \mathrm{MPa}$ and $\Delta E_{b} / c^{1 / 3}=2.58-7.53 \mathrm{eV}[6,7]$ with the larger values for $\mathrm{Cr}$ and $\mathrm{Mn}$, which have very large volume misfits in $\mathrm{Al}$. For basal slip in $\mathrm{Mg}-\mathrm{X}(\mathrm{X}=\mathrm{Al}, \mathrm{Zn})$, we obtain $\tau_{y 0} / c^{2 / 3} \sim 223 \mathrm{MPa}$ and $\Delta E_{b} / c^{1 / 3} \sim 1.92 \mathrm{eV}[12]$ Thus, the fit parameters for the alloys considered here are quite reasonable.

With the fitted quantities, the predictions of flow strength as a function of temperature and composition using the full multiscale bow out model are shown in Fig. 6. The results for $\mathrm{Cu}-1 \% \mathrm{Ge}$ are very slightly underestimated by the model, but in all other cases the predictions are in excellent agreement with the experimental data. Thus, the concentration-normalized energy barrier and zero temperature strength parameters are sufficient to explain the strengthening behavior of these alloys over a wide range of solute concentrations and temperature without the need to invoke any other mechanisms. Also shown in Fig. 6 are the predictions of the low-temperature model The experimental data clearly deviate from the low-temperature model generally around 300K, and the deviation is captured well by the full model. The predictions of the logarithmic model with the 0.55 coefficient are, as noted earlier, above the experiments at low temperature but coincide 
This is an author-created, un-copyedited version of an article accepted for publication/published in Modelling and Simulation in Materials Science and Engineering. IOP Publishing Ltd is not responsible for any errors or omissions in this version of the manuscript or any version derived from it. The Version of Record is available online at http://dx.doi.org/10.1088/0965-0393/24/6/065005. Citation details: Leyson, G. P. M.; Curtin, W. A. Model. Simul. Mater. Sci. Eng. 2016, 24 (6), 65005.

(a)

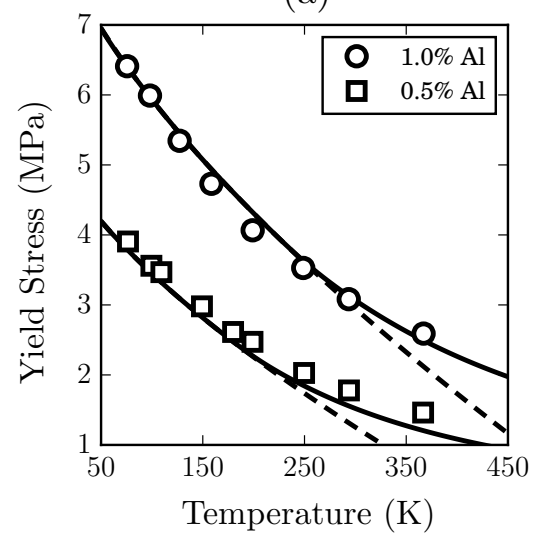

(b)

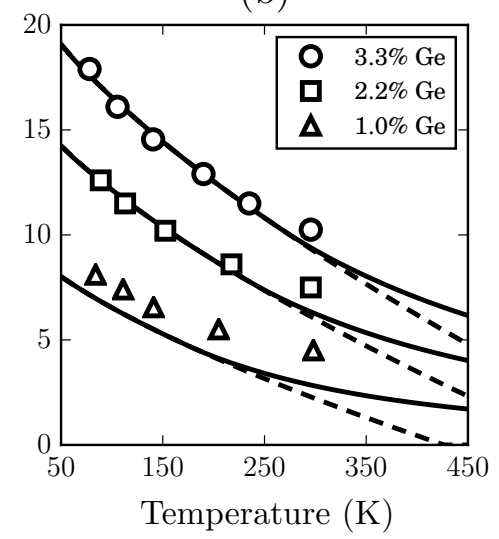

(c)

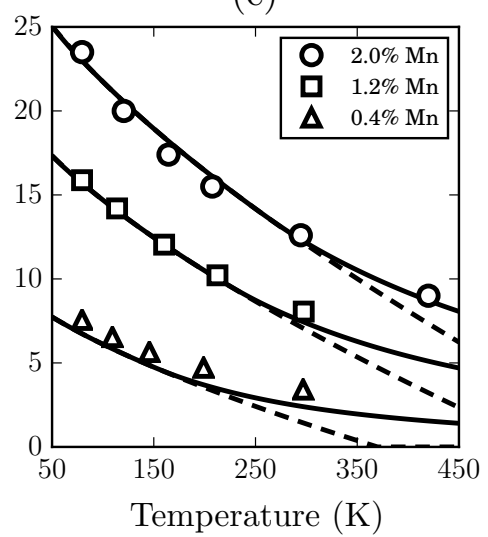

Figure 6: Yield stress versus temperature for various Cu-based alloys: (a) $\mathrm{Cu}-\mathrm{Al}$ [13]; (b) $\mathrm{Cu}-\mathrm{Ge}$ [14]; (c) $\mathrm{Cu}-\mathrm{Mn}$ [14]. Open symbols correspond to experimental measurements, bold solid lines are predictions of the full multiscale bow out model using parameters shown in Table 1, and the dashed lines are predictions using the low-temperature model.

with the full model at the higher temperatures. In these experiments, no transition toward the power-law regime of Eqn.(21)) is evident nor is it expected based on the parameter values.

Further verification of the multiscale bow-out model is seen in the dependence of the activation volume $V$ on the yield stress $\tau_{y}$. Figure 7 shows a log-log plot of the model predictions, using the various approximate models to facilitate identification of transitions in form, along with the experimental measurements for $\mathrm{Cu}-\mathrm{Al}$ at two temperatures [13]. Due to the very low stresses for some of these materials at $298 \mathrm{~K}$, we do account for the low but finite Peierls stress of pure $\mathrm{Cu}$ at $\mathrm{T}=298 \mathrm{~K}$. At the low temperature of $T=78 \mathrm{~K}$, the low-temperature model captures most of the data well, qualitatively and quantitatively, but at the lowest strengths (corresponding to low $\mathrm{Al}$ concentration alloys), there is a clear deviation from the low- $T$ result toward a trend that is well-captured by the logarithmic model. At the higher temperature of $T=298 \mathrm{~K}$, the low- $T$ model is poor over the entire range while the logarithmic model captures the trend well over most of the range, although with a difference in magnitude that is typical of differences found in previous applications of our model to $\mathrm{Al}$ alloys. At this higher temperature, the lowest strength data also starts to show a more-rapid increase in the activation volume with decreasing strength. This coincides with the regime in which the power-law model begins to control the predicted activation volume; while the data is not sufficient to draw a strong conclusion, the emerging trend is qualitatively captured by the present model.

The predicted activation volume versus temperature is also compared with experimental measurements by Wille et al. [14] on the $\mathrm{Cu}-\mathrm{Mn}$ and $\mathrm{Cu}-\mathrm{Ge}$ alloys, as shown in Fig. 8. For both alloy systems, the model predicts the correct scaling behavior of the activation volume at high temperatures (i.e. $\ln V \sim T$ ), clearly improving on the predictions of the low-T results. With trends in strength and activation volume well-predicted by the model, the results of Wille et al. are seen to be fully consistent with solute strengthening and there is no need to introduce possible effects of solute clusters, as pursued by Wille et al., to rationalize the measured behavior. However, the predictions for activation volume are not quantitatively accurate, deviating by factors 
This is an author-created, un-copyedited version of an article accepted for publication/published in Modelling and Simulation in Materials Science and Engineering. IOP Publishing Ltd is not responsible for any errors or omissions in this version of the manuscript or any version derived from it. The Version of Record is available online at http://dx.doi.org/10.1088/0965-0393/24/6/065005. Citation details: Leyson, G. P. M.; Curtin, W. A. Model. Simul. Mater. Sci. Eng. 2016, 24 (6), 65005.

(a)

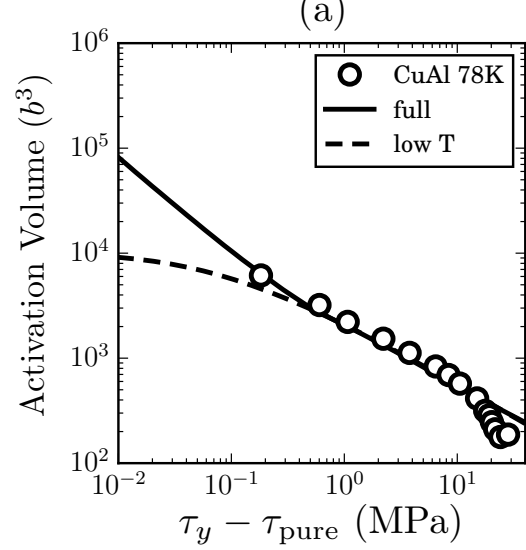

(b)

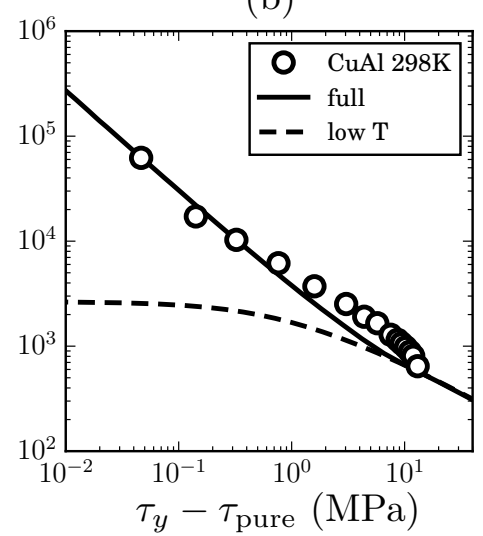

Figure 7: Activation volume $V$ versus yield stress $\tau_{y}$ for $\mathrm{Cu}$ - $\mathrm{Al}$ alloys at (a) $78 \mathrm{~K}$ and (b) $298 \mathrm{~K}$. Open symbols are experimental measurements from Basinski et al. [13]. Solid line and dashed lines correspond to the full multiscale bow out and low temperature model predictions, respectively. $\tau_{\text {pure }}$ is the yield stress of pure $\mathrm{Cu}$.

of $\sim 1.3$ and $\sim 1.6$ for $\mathrm{Cu}-\mathrm{Mn}$ and $\mathrm{Cu}-\mathrm{Ge}$, respectively. Being a derivative of the energy barrier versus stress, the activation volume is much subtle quantity, and deviations between theory and experiment occur even in Al-X alloys. Thus, we emphasize that the model captures the trend $\ln V \sim T$ and defer quantitative differences until more detailed results can be obtained for solute interactions with dislocations in $\mathrm{Cu}$. In fact, the low fitted energy barrier for $\mathrm{Cu}-\mathrm{Ge}$ and the low stacking fault energy of $\mathrm{Cu}$ might suggest that a full analysis would reveal a second solution for the equilibrium configuration (as found for $\mathrm{Mg}-\mathrm{Al}$ and $\mathrm{Mg}-\mathrm{Zn}$ [12]) which might also contribute to the high- $T$ strengthening and activation volume. Again, resolution of these questions awaits more detailed analysis.

\section{Summary}

We have extended our previous single-scale bow-out model for low temperature solution strengthening to account for larger-scale waviness of the dislocations that can take advantage of larger-scale fluctuations in the random solute distributions. As a result of such fluctuations, it is energetically favorable for the dislocation to undergo multiscale bow-out, creating a range of energy barriers that control the thermally-activated dislocation motion at lower stresses or higher temperatures. Our analytic model yields a scaling of the energy barrier versus stress that reconciles different functional forms proposed in the literature, and provides the overarching framework for predicting the domains where the different forms become apparent. We have validated the model using literature data with the two key parameters fit for any given alloy, independent of alloy concentration or temperature. The multiscale bow-out model captures the quantitative and qualitative trends in both yield stress and activation volume measured in the experiments. Thus, the new model provides a complete and predictive framework for solute strengthening over the wide range of experimentally-relevant conditions. 
This is an author-created, un-copyedited version of an article accepted for publication/published in Modelling and Simulation in Materials Science and Engineering. IOP Publishing Ltd is not responsible for any errors or omissions in this version of the manuscript or any version derived from it. The Version of Record is available online at http://dx.doi.org/10.1088/0965-0393/24/6/065005. Citation details: Leyson, G. P. M.; Curtin, W. A. Model. Simul. Mater. Sci. Eng. 2016, 24 (6), 65005.

(a)

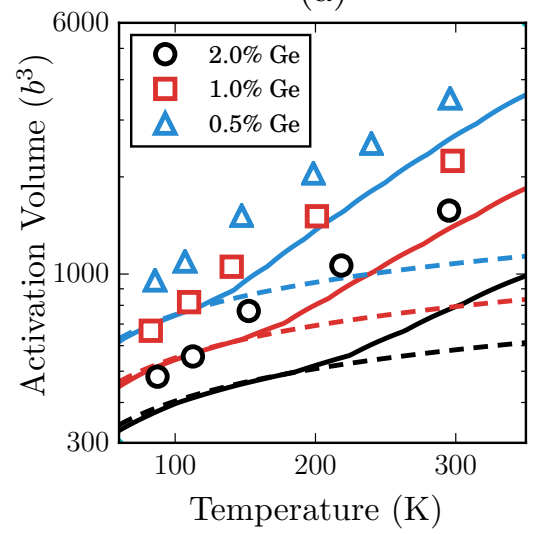

(b)

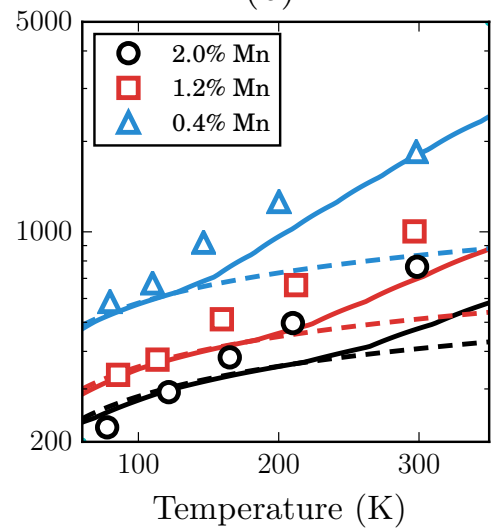

Figure 8: Activation volume $V$ versus temperature $T$ for (a) $\mathrm{Cu}-\mathrm{Ge}$ and (b) $\mathrm{Cu}-\mathrm{Mn}$ alloys at various concentrations. Open symbols are experimental measurements from Wille et al. [14]. Solid line and dashed lines correspond to the full multiscale bow out and low-temperature model predictions, respectively.

\section{References}

[1] Trinkle, D.R., Woodward, C.. The chemistry of deformation: How solutes soften pure metals. Science 2005;310(5754):pp. 1665-1667.

[2] Yasi, J.A., Hector Jr., L.G., Trinkle, D.R.. First principles data for solid-solution strengthening in magnesium: from geometry and chemistry to properties. Acta Materialia 2010;58:5704-5713.

[3] Zander, J., Sandstrom, R., Vitos, L.. Modelling mechanical properties from non-hardenable aluminum alloys. Computational Materials Science 2007;41:86-95.

[4] Zander, J., Sandstrom, R.. One parameter model for strength properties of hardenable aluminum alloys. Materials and Design 2008;29:1540-1548.

[5] Caillard, D., Martin, J.L.. Thermally Activated Mechanisms in Crystal Plasticity. Pergamon; 2003.

[6] Leyson, G.P.M., Curtin, W.A., Hector Jr., L.G., Woodward, C.F.. Quantitative prediction of solute strengthening in aluminum alloys. Nature Materials 2010;9:750-755.

[7] Leyson, G.P.M., Hector Jr, L.G., Curtin, W.A.. Solute strengthening from first-principles and applications to aluminum alloys. Acta Materialia 2012;60:3873-3884.

[8] Labusch, R., Grange, G., Ahearn, J., Haasen, P.. Rate processes in plastic deformation of materials. Cleveland, OH: American Society for Metals; 1975, p. 26.

[9] Zaiser, M.. Dislocation motion in a random solid solution. Philosophical Magazine A 2002;82(15):2869-2883. 
This is an author-created, un-copyedited version of an article accepted for publication/published in Modelling and Simulation in Materials Science and Engineering. IOP Publishing Ltd is not responsible for any errors or omissions in this version of the manuscript or any version derived from it. The Version of Record is available online at http://dx.doi.org/10.1088/0965-0393/24/6/065005. Citation details: Leyson, G. P. M.; Curtin, W. A. Model. Simul. Mater. Sci. Eng. 2016, 24 (6), 65005.

[10] Dong, Y., Nogaret, T., A, C.W.. Scaling of dislocation strengthening by multiple obstacle types. Metallurgical and Materials Transactions A 2010;41A:1954-1960.

[11] Huse, D.A., Henley, C.L.. Pinning and roughening of domain walls in ising systems due to random impurities. Physical Review Letters 1985;54(25):2708-2711.

[12] Leyson, G.P.M., Hector Jr, L.G., Curtin, W.A.. First-principles prediction of yield stress for basal slip in Mg-Al alloys. Acta Materialia 2012;(in press)(60):5197-5203.

[13] Basinski, Z.S., Foxall, R.A., Pascual, R.. Stress equivalence of solution hardening. Scripta Metallurgica 1972;6(9):807-814.

[14] Wille, T.H., Gieseke, W., Schwink, C.H.. Quantitative analysis of solution hardening in selected copper alloys. Acta Metallurgica 1987;35(11):2697-2693. 